\title{
La critica semiologica in Italia
}

\author{
Cesare Segre
}

Non c'è dubbio che sia stata una grande e bella avventura quella dell'affermazione in Italia della critica a base semiotica. Essa rientra fra le innovazioni favorite in Italia dalla fine del regime fascista e dall'attenuazione del predominio crociano nella filosofia e nella letteratura. Attraverso un'imponente attività di traduzione, e subito dopo l'elaborazione originale di idee giunte dall'estero, si sono susseguite le affermazioni della critica stilistica di Spitzer e di Auerbach, poi dei vari tipi di critica sociologica, con i modelli proposti da Lukács, da Goldmann, da Benjamin (di cui s'ignoravano ancora, forse di proposito, i lati mistici e religiosi). Due correnti all'inizio contrapposte, dato che la prima insisteva sulla critica verbale, la seconda sulle interrelazioni letteratura-società. Vennero poi, quasi senza lasciare il tempo per sedimentarsi, la critica strutturalista di Barthes e di Lévi-Strauss, quella strutturalistico-semiologica della scuola di Praga (in particolare di Mukarôvsky) e di Jakobson, la riscoperta del Formalismo russo (人klovskij, Tynjanov, Tomasêvskij) e della narratologia di Propp, subito ripresa e trasformata da Todorov e Bremond, infine, ancora dalla Russia, gli stimoli inesauribili di Lotman e della scuola di Tartu e la rivalutazazione di Bachtin, che parve un luogo d'incontro di spunti formalistici e sociologici. La Francia proponeva ancora la (troppo) rigorosa semiotica di Greimas.

Quadro generale: N. Borsellino, Dallo storicismo al post-strutturalismo. Un trentennio di critica letteraria, ed E. Raimondi, Le poetiche della modernità e la critica letteraria, in E. Cecchi e N. Sapegno (a cura di), Storia della letteratura italiana, vol. X, tomo 1, Milano, Garzanti, 1987; G. Leonelli, La critica letteraria in Italia (1945-1994), Milano, Garzanti, 1994.

Uno svolgimento teorico lento e progressivo si proponeva d'improvviso all'attenzione in contemporanea, spesso in ordine cronologico inverso. Eppure non ci fu nessun fenomeno di epigonismo, anzi i primi esemplari italiani di critica strutturalista, semiologica e formalista, del 1965 (Avalle, Rosiello, Segre) mostrano che l'elaborazione teorica era avvenuta in modo completo e personale. Fatto sta che in Italia erano da tempo in azione tradizioni di studio particolarmente mature, sul cui terreno le nuove metodologie si poterono innestare benissimo. Alludo alla linguistica storica, alla filologia romanza e alla storia della lingua italiana, che posso sintetizzare nei nomi di Benvenuto Terracini, 
Giacomo Devoto e Gianfranco Contini, attivi in tutti e tre questi campi. Queste tradizioni avevano già assimilato a loro tempo Humboldt e Ascoli, Saussure e Jakobson linguista, Bally e Spitzer. In più, la filologia rappresenta già un esempio di alleanza di metodi concentrati sulla formazione e sulla verifica dei testi, sulla loro interpretazione "globale» (linguistica, metrica, retorica, tematica, contenutistica, ecc.) e anche sulla loro eventuale ambiguità. Anche nell'ambito sociologico la pubblicazione dei Quaderni dal carcere di Gramsci propoponeva metodologie e temi di analisi diversi da quelli giunti dall'estero.

Va aggiunta, come caratteristica italiana della critica semiologica, la diffusa avversione per enunciazioni puramente teoriche, il partire sempre da un testo preciso, da un problema concreto. Ciò dipende dalla consapevolezza che elaborazioni di pensiero avvenute nei puri limiti della mente sono vacue, mentre qualunque ipotesi di lavoro dev'essere confrontata con la realtà, e spesso ne esce mutata o almeno affinata. Le enunciazioni teoriche di àmbito italiano non sono affatto rare, ma sono sempre collegate con la propria applicazione.

Il pieno riconoscimento delle nuove metodologie era consacrato dalla creazione di riviste come «Strumenti critici» (1966) e "Lingua e stile» (1967), e nel 1970 dalla fondazione della Associazione Italiana di studi semiotici, probabilmente la prima associazione nazionale di semiotica. Vi si affiancava attivamente il Centro Internazionale di Semiotica e Linguistica di Urbino, con stages annuali nel mese di luglio. Inutile dire che l'elaborazione teorica e l'applicazione al cinema, ai mass media, ai fumetti, all'antropologia e alla letteratura di consumo seguirono strade non sempre coincidenti con quelle della critica: appunto perché in questi ultimi casi mancava la precedente elaborazione della filologia e della linguistica. E quanto alla linguistica va ricordato, perché in parte attratto da problemi comuni, come quello del testo, della coerenza discorsiva, del discorso riportato, lo sviluppo della corrente generativo-transformazionale e, in rapporto con essa, della Linguistica testuale.

Potrei offrire qui, come in un bollettino di guerra, un elenco delle letterature prese in oggetto (in particolare quelle italiana, inglese, spagnola, francese), o meglio ancora del singoli autori, oppure di tutti i problemi affrontati. Sarebbe una, forse non inutile ma lunghissima, bibliografia ragionata. Preferisco, per brevità, accennare ad alcuni principi generali comuni, se non a tutti i semiologi italiani, almeno alla maggioranza. Ogni gruppo di osservazioni sarà seguito da una bibliografia essenziale.

1. Attenzione alla storia. Ogni procedimento ha una sua storicità, e soprattutto la lingua, strumento unico della letteratura, è interpretabile soltanto tenendo conto dei suoi sviluppi, delle sue stratificazioni, dei suoi registri, dei rapporti con i dialetti o con le altre lingue, ecc. Ciò anticipa le ricerche di stampo bachtiniano, e insieme corrisponde alla nota contemporaneità, sensibile in Italia, di modelli linguistici di diversa datazione, e di «italiani regionali». Pure l'analisi narratologica ha avuto sviluppi storiografici, nel confronto fra le strutture narrative comparate dei testi e delle loro fonti: esempi più ampi gli studi sui rapporti tra Lope de Vega e Boccaccio, e 
quelli sull'uso di fonti cronistiche nei drammi storici di Shakespeare. Sull'esistenza di schemi narratologici impiegati dal lettore nelle sue stesse analisi narratologiche inconsce, ho fatto esperimenti molto promettenti. Altro àmbito storicizzabile affine, perché tocca ancora il problema delle fonti, è lo studio dell'intertestualità, che ha rinnovato l'impostazione dei raffronti minuti fra i testi.

Esempi: M. Pagnini, Critica della funzionalità, Torino, Einaudi, 1973²; A. Ruffinatto, Semiotica ispanica. Cinque esercizi, Alessandria, Edizioni dell'Orso, 1985; Id., Sobre textos y mundos (Ensayos de filología y semiótica hispánicas), Universidad de Murcia, 1989; A. Serpieri et al., Nel laboratorio di Shakespeare, Parma, Pratiche Editrice, 1989; D.S. Avalle, Dal mito alla letteratura e ritorno, Milano, il Saggiatore, 1990; C. Segre, Semiótica filológica (Texto y modelos culturales), Universidad de Murcia, 1990; Id., Intrecci di voci. La polifonia nella letteratura del Novecento, Torino, Einaudi, 1991. Vedi anche: C. Di Girolamo e I. Paccagnella (a cura di), La parola ritrovata. Fonti e analisi letteraria, Palermo, Sellerio, 1982.

2. La concezione della struttura invalsa tra noi è di tipo realistico piuttosto che razionalistico. Di qui la differenza rispetto alla semiotica francese; di qui la preferenza per il metodo induttivo nei confronti di quello deduttivo, e il rifiuto per qualunque modello astratto e astorico, come il «carré sémiotique» di Greimas, o come il catalogo chiuso dei personaggi usato correttamente da Propp per il suo corpus di fiabe, ma generalizzato impropriamente da altri critici, che hanno cercato infelicemente di applicarlo a testi letterari, senza sottoporli alla stessa analisi funzionale usata da Propp. Lo stesso si dica per qualsiasi catalogo chiuso di azioni, attanti e simili.

Esempi: M. Pagnini, Semiosi. Teoria ed ermeneutica del testo letterario, Bologna, il Mulino, 1988; C. Segre, Principios de análisis del texto literario, Barcelona, Crítica, 1985; G.P. Caprettini, Semiologia del racconto, Bari, Laterza, 1992.

3. Attenzione al testo, come prodotto linguistico a più dimensioni, nel quale vengono sintetizzate tendenze epocali e punti di vista, tradizioni e rotture culturali, topoi e temi verbali, ecc., senza trascurare l'ipodiscorso che viene sviluppato con il discorso, e che travalica le partizioni sintattiche, talora integrando, o persino correggendo, il discorso esplicito. Del testo veniva già affrontata la genesi negli studi sulle varianti di redazione (critica delle varianti); ora il discorso è stato ampliato al confronto fra redazioni multiple, e al confronto tra microtesti uniti a formare un macrotesto (raccolta di poesie o di novelle, e simili), nei rapporti con le redazioni precedenti.

Esempi: C. Segre, Sistema y estructura en las «Soledades» de A. Machado, in Id., Critica bajo control, Barcelona, Planeta, 1970² Id, Principios de análisis cit; S. Agosti, Il testo poetico. Teoria e pratiche d'analisi, Milano, Rizzoli, 1972; Id., Critica della testualità, Bologna, il Mulino, 1994; M. Corti, Testo 
o macrotesto? I racconti di Marcovaldo di I. Calvino, in Id., Il viaggio testuale, Torino, Einaudi, 1978; G. Sasso, Le strutture anagrammatiche della poesia, Milano, Feltrinelli, 1982; Id., La mente intralinguistica. L'instabilità del segno: anagrammi e parole dentro le parole, Genova, Marietti, 1993.

4. Il testo è inserito in uno schema comunicativo. Il rapporto emittente-testodestinatario permette di dare il giusto peso sia all'autore, che ha formulato il testo ed è garante della sua potenzialità significativa, sia al destinatario, che intepreta il testo a distanza di tempo e/o di luogo, con inevitabili distorsioni che però egli si sforza di limitare. La lotta alle distorsioni si sviluppa nell'interpretazione del testo, il quale contiene in sé le «istruzioni» che avviano a una lettura fedele. In questo modo si sono evitati i pericoli di una critica reader-oriented, che non si preoccupa dell'emittente e del testo, e quelli di una critica strutturalista pura, che analizza il testo prescindendo da chi lo ha formulato e ignorando l'intervento della "distanza epistemica» tra emittente e destinatario. Si deve comunque tener conto da un lato che il tempo trasforma le potenzialità comunicative del testo, dall'altro che le distorsioni nell'interpretazione sono lo scotto che il testo paga alla propria sopravvivenza. Non va naturalmente taciuto che la comunicazione testuale ha caratteristiche proprie, dato che il testo ingloba in sé il proprio contesto storico, e che d'altra parte, a differenza della comunicazione quotidiana, quella testuale è one way, cioè non prevede feed-back tra emittente e destinatario.

Esempi: M. Pagnini, Le voci dell'ermeneutica e il silenzio dei testi, in "L'Asino d'oro», II, 1991, n. 3; C. Segre, Notizie dalla crisi, Torino, Einaudi, 1993.

5. Il concetto semiotico di segno, in un'analisi approfondita, esorbita necessariamente dalla biunivocità saussuriana significante-significato. Nella letteratura, e nelle arti in genere, il segno in qualche misura viene "creato", perché ha sempre luogo qualche innovazione sia nel valore immediato del singolo segno, sia nello sviluppo di significazione prodotto dal discorso. Il discorso stesso va poi esaminato anche in una prospettiva prelinguistica, data l'esistenza di alternative tra narrazione figurata o verbale, tra immagini e iconismo della parola, e così via.

Esempi: P. Pugliatti e R. Zacchi, Terribilia meditans. La coerenza del monologo interiore in "Ulysses», Bologna, il Mulino, 1983; C. Segre, Linguistica e semiotica, in Id. (a cura di), Intorno alla linguistica, Milano, Feltrinelli, 1983; G. Bottiroli, Retorica della creatività, Torino, Paravia, 1984; Id., Interpretazione e strategia, Milano, Guerini e Associati, 1987; M. di Fazio (a cura di), Narrare: per corsi possibili, Ravenna, Longo, 1989.

6. Lo studio sulle prospettive della narrazione (punto di vista) è stato integrato utilmente nella concezione polifonica di Bachtin, compresa l'attenzione ai vari stili dei parlanti, o anche del pensiero dei parlanti, agli ideologemi e 
al plurilinguismo o alla plurivocità. Sono stati messi in rilievo vari problemi trascurati da Bachtin, come la differenza tra intertestualità (interferenza tra testi letterari individui) e interdiscorsività (utilizzazione di espressioni e sintagmi elaborati in forma collettiva e anonima). Si è pure avanzato molto sulla linea indicata da Ducrot, per l'analisi del discorso riportato.

Esempi: N. Pasero (a cura di), Saggi su Bachtin (con bibliografia), "L'Immagine riflessa», VII, 1984, n. 1/2; G. Ferroni (a cura di), Il dialogo. Scambi e passaggi della parola, Palermo, Sellerio, 1985; B. Mortara Garavelli, La parola d'altri. Prospettive di analisi del discorso, Palermo, Sellerio, 1985; F. Corona (a cura di), Bachtin teorico del dialogo, Milano, F. Angeli, 1986; C. Segre, Intrecci di voci cit.

7. Il problema dei generi letterari è stato in qualche modo unificato mediante la definizione in termini comunicativi dei tipi di testi. Tra diègesi, autobiografia, teatro, lirica, ecc., si riesce a individuare dei modelli comunicativi formalizzabili ed estensibili anche ad altri problemi (per esempio quello del "teatro nel teatro", da Shakespeare a Pirandello). In qualche caso è stato inserito nell'analisi il criterio costituito dallo sfasamento fra tempo narrato e tempo della narrazione.

Esempi: A. Canziani et al., Come comunica il teatro: dal testo alla scena, Milano, il Formichiere, 1978; M. De Marinis, Semiotica del teatro, Milano, Bompiani, 1982; C. Segre, Teatro e romanzo, Torino, Einaudi, 1984; R. Rutelli, Dialoghi con il testo, Napoli, Liguori, 1985.

8. Per i rapporti fra testo e contesto, si sono tratti molti stimoli dalla culturologia di Lotman. Non è parsa accettabile, da Lotman, la tipologia delle culture, talora forzata e priva di spiegazioni sui passaggi da un tipo all'altro. E'invece risultata proficua l'ipotesi di un'omologia tra emergenze di tipo simbolico nella vita quotidiana e il loro trattamento in letteratura. Si è anche tentato di abbozzare un parallelismo fra gli strati più profondi e quelli più superficiali del testo da un lato, e gli strati più profondi e a lento sviluppo, e quelli più superficiali e velocemente mutabili della cultura. Vanno poi ricordate le applicazioni della pragmatica linguistica all'analisi del testo.

Esempi: M. Corti, Ideologie e strutture semiotiche nei «Sermones ad status» del sec. XIII, in Id., Il viaggio testuale, Torino, Einaudi, 1978; C. Segre, Semiótica, historia y cultura, Barcelona-Caracas-México, Ariel, 1981; S. Salvestroni, Semiotica dell'immaginazione, Venezia, Marsilio, 1984; F. Ravazzoli, Il testo perpetuo, Milano Bompiani, 1991.

Se queste sono le linee principali, almeno a mio avviso, della critica semiologica italiana (e sulla loro base ho potuto fornire un repertorio metodologico sistematico dei procedimenti analitici: cfr. il già citato Principios de análisis del texto literario), mi pare interessante aggiungere che essa ha assunto per un certo tempo una funzione di punto d'incontro e di elaborazione anche per altre ten- 
denze critiche, in particolare per quella sociologica e per quella psicoanalitica. Numerosi i saggi in cui queste tendenze vengono espresse con un linguaggio semiologico.

Esempi: A. Serpieri, T.S. Eliot: le strutture profonde, Bologna, il Mulino, 1973; Id., Retorica e immaginario, Parma, Pratiche, 1986; F. Orlando, Illuminismo e retorica freudiana, Torino, Einaudi, 1982; Id, Due letture freudiane: Fedra e il Misantropo, Torino, Einaudi, 1990; S. Agosti, Cinque analisi. Il testo della poesia, Milano, Feltrinelli, 1982; R. Ceserani, Argilla, Napoli, Guida, 1975.

Viceversa il solido fondamento storico e teorico della semiotica italiana ci ha resi quasi impermeabili alle lusinghe del decostruzionismo, mentre ci ha fatto inquadrare in una corretta prospettiva storica la teoria della ricezione, respingendo comunque l'utilizzazione estetica della ricezione, che si allea all'impianto irrazionalistico e talora sofistico del decostruzionismo.

Storia e critica della ricezione: M.L. Meneghetti, Il pubblico dei Trovatori. La ricezione della poesia cortese sino al XIV secolo, Torino, Einaudi, 1992.

Ora qualcuno dice che la critica semiologica è in crisi; in verità la crisi investe tutta la critica. Si tratta di una malattia ancora mal nota, perciò difficile da diagnosticare. Tutta la storia della cultura è fatta di movimenti (e movimenti letterari) che vengono scalzati da altri, magari quando sembravano ancora pieni di promesse: basta pensare al passaggio dall'erudizione del «metodo storico" alla critica estetica di matrice idealistica. Ma nel nostro caso non abbiamo a che fare con questo fenomeno.

Per capire meglio, è intanto utile rilevare il diverso funzionamento delle cose della cultura in Francia e in Italia. In Francia, proprio perché la cultura ha un maggior impatto sulla quotidianità e sulla gente comune, essa ricorre quasi necessariamente a teatralizzazioni e colpi di scena: successi vittoriosi e cadute più o meno rovinose, contrapposizioni e alternanze partecipate passionalmente dal pubblico e, in senso proprio o figurato, fortemente politicizzate. Dato fondamentale poi la personalizzazione: ogni movimento s'incarna in uno o più portabandiera, ed è legato alle loro fortune. La critica strutturalisticosemiologica fa dunque tutt'uno con personaggi la cui morte (Foucault, Barthes, Greimas) o il cui passaggio ad altre attività e altri interessi (Kristeva, Todorov, Bremond) ha praticamente segnato il declino del movimento.

In Italia tutto va (o almeno è andato in questo caso) in modo più ponderato e tranquillo. E infatti i critici italiani non hanno esaltato i nuovi metodi come scoperte rivoluzionarie ed esclusive, ma li hanno semplicemente considerati strumenti utili per approfondire la descrizione e la comprensione dei testi letterari. Questa mancanza di dogmatismo, questo atteggiamento prevalentemente operativo, ha permesso di assimilare molte delle proposte diffuse o rese note in séguito. Ora in molti paesi si tende a parlare di post-strutturalismo, alludendo all'abbandono o al diverso trattamento di elementi tipici dello strutturalismo (lo studio immanente dei testi, l'entusiasmo per l'analisi delle 
funzioni) e della semiotica, in particolare di Greimas (il «carré sémiotique», le modalità, ecc.). Si potrebbe dire, in base a quanto appena notato, che in Italia lo strutturalismo è nato come post-strutturalismo, sicché il cambio di prospettive e di proporzioni si trova già messo in atto senza troppo rumore. Lo stesso vale per la Scuola russa di Tartu e Mosca, come la nostra fondata su basi filologiche (si risale sino a Potebnja e Veselovskij), come la nostra più storicizzante che razionalizzante. Ho parlato prima, appunto, di impianto realista, che abbiamo in comune con gli studiosi russi.

Scarso dogmatismo, apertura a nuovi stimoli, duttilità, legami con la tradizione, sono caratteristiche che avrebbero potuto assicurare un sereno avvenire alla nostra critica. E, se in vena di ottimismo, sarebbe stato persino lecito affermare, specie dopo la fecondissima assimilazione del pensiero di Bachtin, che non ha più senso parlare di critica strutturalistica o semiologica, solo perché ormai è in opera una critica che, pur nata su quel terreno, va molto al di là dei presupposti fondanti. E a questa critica non si possono porre limiti di tempo.

E tuttavia la crisi c’è, e si avverte. Cercherò di dare qualche concretezza al fantasma. Non si deve tacere, prima di tutto, la crisi delle ideologie e dei valori in cui ci hanno precipitato gli avvenimenti politici degli ultimi anni. Direttamente o indirettamente, alle ideologie ora screditate o contestate si faceva riferimento; e il quadro generale di convinzioni e impegni era (sembrava) assodato. $E^{\prime}$ in corso un rimescolamento da cui si dovrà uscire prima o poi, ma che per ora porta confusione e incertezza. In questo quadro, è difficile azzardare nuove elaborazioni in qualunque campo del sapere umanistico, perciò anche in quello della critica. Di qui, quanto meno, un'impressione di stasi. La crisi è generale, investe anche la critica, non solo la critica.

Non va dimenticato, per contrasto, il fatto che le nuove metodologie critiche si erano affermate da noi contestualmente e successivamente ai fermenti del '68, al boom dell'editoria saggistica, e in particolare linguistica, alla fondazione e alla fortuna di riviste impegnate a diffondere le idee più stimolanti in ambito di lingua e letteratura. Ora domina il riflusso, idee nuove non se ne presentano molte, e si diffonde la rassegnazione di fronte a un mondo a cui l'intelligencija si sente giustamente estranea.

Si pone infatti al di fuori della contingenza un rimescolamento dei rapporti di prestigio conseguente al tipo di cultura imposto dal neocapitalismo attraverso la civiltà multimediale. L'umanesimo che ha fatto, bene o male, da supporto a tutti i movimenti letterari, è ora in posizione di difesa, se non di regresso. La letteratura ha un prestigio sempre più scarso tra le molte e rumorose offerte del mondo attuale. La critica letteraria, che non è altro che l'interprete e la celebratrice della letteratura e delle sue funzioni edonistiche ma anche gnoseologiche, suggestive ma anche stimolatrici di smascheramento e di rinnovamento, è portata a declinare col declino della letteratura stessa.

Da queste riflessioni (per non dire constatazioni) scaturisce la necessità di riformulare il concetto di valore letterario. Già la stessa idea di letteratura si 
trasforma attraverso il tempo: opere considerate in origine come non letterarie sono poi state annesse alla letteratura, e inversamente sono state espunte dalla letteratura opere considerate dapprima di sua pertinenza. Anche i valori mutano col mutare dei paradigmi usati per misurarli. E' giusto considerare punto di arrivo della critica il giudizio di valore, purché si tenga presente che un valore è tale solo in rapporto con i paradigmi epocali o personali. Il valore che permane al di là delle vicende del gusto è quello costituito: I) dalla capacità di un'opera di sintetizzare la visione del mondo di un'epoca, di essere, per dirla con la Scuola di Tartu, un "modello del mondo»; II) dal suo potere di smascheramento e di proposta, verificabile attraverso l'impatto dell'opera con la società e con la coscienza contemporanee, e nei casi più eccellenti anche con quelle successive.

Fra le necessità più urgenti della critica, va posta poi quella di far riferimento a un'estetica. Esaminare com'è fatta, in tutti i sensi, un'opera letteraria, dev'essere operazione iniziale e imprescindibile di un'analisi che punta verso un altro problema: come agisce l'opera sul lettore, e quali criteri egli può usare per rendersi conto di questa azione? Pare curioso che questo problema sia scarsamente sentito, anche se è nello stesso tempo innegabile che l'estetica, in una fase di scarsa vitalità, non pare promettere un grande aiuto.

Se la crisi della critica sussiste davvero, è perché si sono fatti rari o deboli i tentativi di soluzione, e si fatica a formulare nuovi piani di lavoro. Non c'è motivo di arrendersi (e a chi?), ma piuttosto di riflettere e approfondire, con serena operosità, in attesa di nuovi entusiasmanti stimoli, se verranno. A costo di essere accusato di moralismo, dirò che considero più utili esercizi e tentativi condotti col massimo impegno, che dibattiti astratti. La letteratura è una cosa seria: cerchi di esserlo anche la critica. Bisogna però aggiungere che il nostro lavoro, come qualunque lavoro intellettuale, ha bisogno di svolgersi in un ambiente politicamente e moralmente sano; questo purtroppo, oggi, in Italia non si dà, anche se molti cercano di resistere e di salvare i valori più alti. In qualche modo la lotta in difesa della critica è anche la lotta in difesa della democrazia. 